\title{
Weiterbildungsqualität statt Zusatzzertifikate
}

\author{
Luzia Birgit Gisler, Insa Koné, Regula Kronenberg, Julia Laukenmann, Gabriela Rohrer, Manuel Schaub, \\ Deborah Schneider
}

aus dem Think Tank Politics der Jungen Hausärztinnen und -ärzte Schweiz (JHaS)

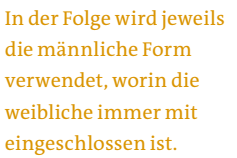

eingeschlossen ist.
Korrespondenz:

Dr. med. Regula Kronenberg JHaS Think Tank Politics CH-3000 Bern

regula.kronenberg[at]jhas.ch
In der Schweizerischen Ärztezeitung Nr. 11, 2018, erschien als Themenschwerpunkt eine Diskussion zum Weiterbildungsprogramm Allgemeine Innere Medizin. Im ersten Artikel [1] schreibt Herr Dr. Marty, dass sich

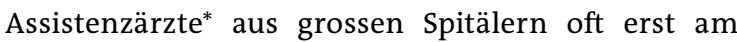
Schluss der Weiterbildung für eine Praxisassistenz bewerben. Dabei erkennen sie konkrete Defizite in ihren Kompetenzen, die sie nur mit Mühen wettmachen können, so dass sie sich unter Umständen gegen eine Laufbahn als Hausärzte entscheiden. Zudem berichtet Dr. Marty, dass der Weg zum Facharzttitel Allgemeine Innere Medizin für Ärztinnen mit Kleinkindern in der Regel versperrt bleibt trotz guter interdisziplinärer Weiterbildung wegen fehlender Teilzeitarbeitsmöglichkeiten in A-Kliniken. Im zweiten Diskussionsbeitrag [2] beschreibt Herr Dr. Sorg die Wichtigkeit von Praxisassistenzen und fordert zwei Jahre Praxisassistenz zu Beginn der Weiterbildung.

Dr. Sorg [2] und Dr. Heim [3] sorgen sich ausserdem um die Kenntnisse am Bewegungsapparat für eine kompetente traumatologische Grundversorgung. Gemäss Dr. Heim könnten die zunehmende Spezialisierung, das immer grösser werdende Fachspektrum und das fehlende Angebot, diese chirurgischen Tätigkeiten zu erlernen, Gründe dafür sein.

In derselben Ausgabe der Schweizerischen Ärztezeitung gibt der Präsident des Schweizerischen Instituts für ärztliche Weiter- und Fortbildung, Herr Dr. Bauer [4], zu bedenken, dass Ärzte in Weiterbildung immer weniger Zeit am Krankenbett und im Gespräch mit den Patienten verbringen, jedoch immer länger am Computer und am Telefon arbeiten. Herr Prof. Bassetti und Herr Dr. Héritier [5] erwähnen in der Replik die Empfehlungen der "Arbeitsgruppe Nachwuchsförderung", die unter dem Motto «More action, less administration» auf eine Reduktion von administrativen Tätigkeiten hinzielen.

Wir unterstützen diese Beobachtungen und Voten und fordern entsprechende Massnahmen:

1) Förderung früher Praxisassistenzen zu Beginn der Weiterbildung. Dazu braucht es entsprechende Finanzierung sowie ein gezieltes Coaching der jungen
Ärzte in Weiterbildung. Dadurch wird einerseits früh der Grundstein für den Berufswunsch Hausarzt gelegt, und andererseits erkennt der Assistenzarzt früh, welche Fähigkeiten er sich in seiner Weiterbildung erwerben muss, und kann die Weiterbildungsstellen entsprechend planen.

2) Einfacherer Zugang zu A-Kliniken inklusive Teilzeitarbeit. Lehrärzte und Institute für Hausarztmedizin sollen vermehrt mit A-Kliniken zusammenarbeiten. Es braucht mehr kombinierte Weiterbildungsangebote und Teilzeitstellen, damit sowohl Eltern wie auch Quereinsteiger mit breiter klinischer Erfahrung einfacher zum "A-Jahr» in Innerer Medizin kommen. Damit kann viel menschliches Potenzial, das aktuell noch brachliegt, zugunsten der Hausarztmedizin ausgeschöpft werden.

3) Motivierte, hochstehende Lehrkultur; zurück ans Patientenbett, weg vom PC. Personelle und finanzielle Ressourcen müssen wieder vermehrt investiert werden in die Begleitung von Assistenzärzten beim Erlernen praktischer Fertigkeiten. Teaching beim Untersuchen, Punktieren, Gipsen usw. stehen aktuell leider zu sehr hinter dem grossen administrativen Aufwand an.

4) Kampf dem Zertifizierungswahn. Die Weiterbildungszeit soll nicht verlängert und durch unzählige obligatorische zeit- und kostenintensive Zusatzkurse und -zertifikate verteuert werden. Die gefragten praktischen Fähigkeiten sollen vielmehr im direkten Teaching innerhalb der Weiterbildungszeit erlernt werden können.

\section{Literatur}

1 Marty F. Sechs Jahre Weiterbildungsprogramm "Allgemeine Innere Medizin». Ein Feedback aus der Praxis. Schweiz Ärztezeitung. 2018;99(11):353-5.

2 Sorg B. Defizite in der Ausbildung von Hausärztinnen und Hausärzten. Fehlende Kompetenz am Bewegungsapparat. Schweiz Ärztezeitung. 2018;99(11):356.

3 Heim D. Replik auf den Beitrag von Dr. B. Sorg. High Noon Schweiz Ärztezeitung. 2018;99(11):359.

4 Bauer W. Reden ist Silber, Handeln wäre Gold ... Schweiz Ärztezeitung. 2018;99(11):364.

5 Bassetti S, Héritier F. Replik auf die Beiträge von Dr. F. Marty und Dr. B. Sorg. Wir brauchen ein möglichst flexibles Weiterbildungsprogramm. Schweiz Ärztezeitung. 2018;99(11):357-8. 